\title{
Data regeneration
}

\author{
A regenerator capable of simultaneously removing both phase and amplitude noise from an optical \\ data stream may be a critical component of future optical networks. Radan Slavík explained to \\ Nature Photonics how he and his co-workers realized such a device.
}

Tell us about your work.

We have built the first practical regenerator capable of suppressing both amplitude and phase noise in binary phase-encoded optical communications signals. The device takes an incoming noisy data signal and restores its fidelity, therefore acting as a true 'black box' signal regenerator. This is of acute importance for high-spectral-efficiency advanced modulation formats, which are strongly compromised by phase noise originating from nonlinear interactions with other wavelength channels travelling along the same optical fibre. In our work, phase and amplitude noise removal was successfully demonstrated at data rates of $10 \mathrm{Gbit} \mathrm{s}^{-1}$ and $40 \mathrm{Gbit} \mathrm{s}^{-1}$, but our device can in principle handle much higher data rates, limited only by the available pump wavelength separation and dispersion control within the nonlinear fibre. Our research potentially represents a basic building block for signal regeneration systems that handle multilevel phaseand amplitude-encoded signals capable of carrying much more than one bit per symbol.

What is the physical mechanism behind the regenerator?

The core element is a phase-sensitive amplifier, which relies on the interaction between a weak, noisy incoming signal beam and two stronger 'clean' pump beams of different wavelengths in a highly nonlinear optical fibre. Stabilizing the optical phase relationship between the signal and pump beams allows the signal to inherit the 'clean' phase characteristics of the pumps, and thus be regenerated. The phase-sensitive amplifier selectively amplifies signals with a phase of 0 or $\pi$, while attenuating signals with a phase of $\pi / 2$ and $3 \pi / 2$. Thus, the real part of the signal electric field, where the noiseless data is originally found, is amplified, whereas the imaginary part, which is generated by noise, is attenuated. This mechanism inherently reduces the phase noise, but in general causes the phase noise to be transferred to amplitude noise, which is undesirable. We therefore used the phase-sensitive amplifier in saturation mode, which

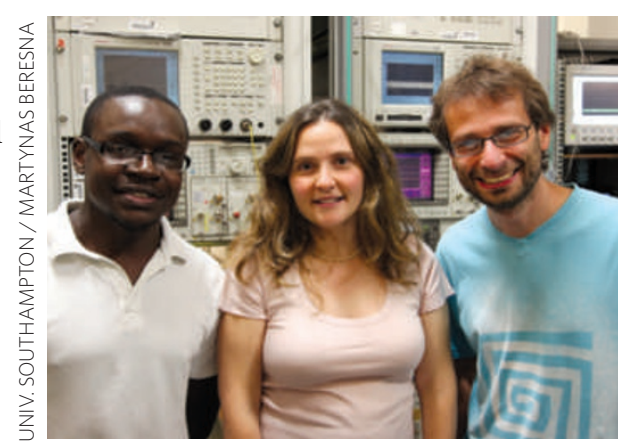

Left to right: Joseph Kakande, Francesca Parmigiani and Radan Slavík.

requires a relatively high input signal power with respect to the pumps, to suppress the amplitude noise. Besides suppressing the phase-to-amplitude noise transfer, it also reduces the amplitude noise present in the incoming data.

\section{What were the challenges in constructing your device?}

The main challenge in realizing a practical system was the phase stabilization of multiple waves at different wavelengths within the amplifier. In previous schemes this was realized in an 'artificial' way: all of the phase-locked optical waves required were generated prior to the creation of the signal beam. This is unsuitable for use in a real transmission system, in which only the data signal is transmitted, not the pump beams. Furthermore, using electro-optic modulation to generate the phase-locked beams restricts the maximum data rate of the signals, which again is not ideal. Instead, we implemented a two-step alloptical method. First, we used a nonlinear four-wave mixing process to recover the optical carrier from the incoming noisy binary phase-encoded signal. A narrowbandwidth continuous-wave laser serving as pump 1 was mixed with the noisy signal, and the carrier was generated through a degenerate four-wave mixing process in a highly nonlinear fibre. Unfortunately, during this process any noise in the original data signal is transferred to the carrier, which also generally suffers from a relatively low optical signal-to-noise ratio. Thus, in the second step we exploited injection-locking of a semiconductor laser to improve the optical signal-to-noise ratio and clean the noise from the recovered carrier, which then served as pump 2 . The phase-synchronized pump 1 and pump 2 served as the two strong 'clean' pump beams in the subsequent phase-sensitive amplification process. The combined all-optical processes allow us to realize a compact ultrafast phase synchronization scheme that enables 'black-box' operation of the regenerator.

\section{What are the potential applications?} The device is largely targeted for use in fibre-optic communications, where it may enable more data channels to be transmitted along the same fibre or allow longer distances between repeaters. However, there are many other emerging applications in which such devices could prove invaluable, such as in all-optical signals processing. Our device can be viewed as a one-bit analog-to-digital convertor, and may be used as a basic building block for more complicated circuits in this field. Other applications are likely to arise in quantum communications, metrology and potentially even free-space ultralong-haul communications.

\section{What are your future plans?}

Along with making our current regenerator more robust for use over a real fibre-optic network, we are looking at making it capable of handling more complex modulation formats that have higher spectral efficiencies. Indeed, we are now working on extending the technology to the regeneration of quadrature phase-shift-keyed format signals in which the information is encoded in four phase levels $(0, \pi / 2, \pi$ and $3 \pi / 2)$ rather than two, as in current systems. This would allow the transmission of two bits per symbol and thus should allow a twofold increase in network capacity.

\section{INTERVIEW BY RACHEL WON}

Radan Slavík and his co-workers have a Letter describing their all-optical phase and amplitude regenerator on page 690 of this issue. 